\title{
Hodge theory on nearly Kähler manifolds
}

\author{
Misha VERBitSKY
}

Let $(M, I, \omega, \Omega)$ be a nearly Kähler 6-manifold, that is, an $\mathrm{SU}(3)$-manifold with $(3,0)$-form $\Omega$ and Hermitian form $\omega$ which satisfies $d \omega=3 \lambda \operatorname{Re} \Omega, d \operatorname{Im} \Omega=-2 \lambda \omega^{2}$ for a nonzero real constant $\lambda$. We develop an analogue of the Kähler relations on $M$, proving several useful identities for various intrinsic Laplacians on $M$. When $M$ is compact, these identities give powerful results about cohomology of $M$. We show that harmonic forms on $M$ admit a Hodge decomposition, and prove that $H^{p, q}(M)=0$ unless $p=q$ or $(p=1, q=2)$ or $(p=2, q=1)$.

\section{Introduction}

\subsection{Nearly Kähler 6-manifolds}

Nearly Kähler manifolds (also known as $K$-spaces or almost Tachibana spaces) were defined and studied by Alfred Gray $[11 ; 12 ; 13 ; 14]$ in the general context of intrinsic torsion of $U(n)$-structures and weak holonomies. An almost complex Hermitian manifold $(M, I)$ is called nearly Kähler if $\nabla_{X}(I) X=0$, for any vector field $X$ ( $\nabla$ denotes the Levi-Civita connection). In other words, the tensor $\nabla \omega$ must be totally skew-symmetric for $\omega$ the Hermitian form on $M$. If $\nabla_{X}(\omega) \neq 0$ for any nonzero vector field $X$, then $M$ is called strictly nearly Kähler.

Using deep results of Kirichenko [21] and Cleyton and Swann [7], P-A Nagy [26] has shown that any strictly nearly Kähler manifold is locally a product of locally homogeneous manifolds, strictly nearly Kähler 6-manifolds, and twistor spaces of quaternionic Kähler manifolds of positive Ricci curvature, equipped with the EelsSalamon metric [8].

These days the term "nearly Kähler" usually denotes strictly nearly Kähler 6-manifolds. We shall follow this usage, often omitting "strictly" and "6-dimensional". In more recent literature (such as Mororianu, Nagy and Semmelmann [24]), these objects are called Gray manifolds. 
For a history of this notion, a number of equivalent definitions and a bibliography of current work in this field, we refer the reader to Mororianu, Nagy and Semmelmann [23] and our paper [31].

It is convenient to define nearly Kähler 6-manifolds in terms of differential forms, as follows.

Proposition 1.1 Let $(M, I, \omega)$ be a Hermitian almost complex 6-manifold. Then the following conditions are equivalent.

(i) The tensor $\nabla_{X}(I) Y$ is skew-symmetric with respect to $X, Y$, and is nonzero.

(ii) The form $\nabla \omega \in \Lambda^{1}(M) \otimes \Lambda^{2}(M)$ is nonzero and totally skew-symmetric. Notice that in this case, by Cartan's formula, we have $d \omega=\nabla \omega$.

(iii) There is a $(3,0)$-form $\Omega$ with $|\Omega|=1$, and

$$
\begin{aligned}
d \omega & =3 \lambda \operatorname{Re} \Omega, \\
d \operatorname{Im} \Omega & =-2 \lambda \omega^{2},
\end{aligned}
$$

where $\lambda$ is a nonzero real constant.

Proof Refer to Gray [12]; see also Baum et al [5] or Verbitsky [31, Theorem 4.2].

Definition 1.2 An SU(3)-manifold $(M, \omega, \Omega, I)$ is called nearly Kähler if (1-1) holds.

The examples of compact nearly Kähler manifolds are scarce; one may hope that the nearly Kähler orbifolds would occur more often. The results of this paper are stated for manifolds, but they are valid for all nearly Kähler orbifolds, with the same proofs.

The most puzzling aspect of nearly Kähler geometry is a complete lack of nonhomogeneous examples. With the exception of 4 homogeneous cases described below (Section 1.2), no other compact examples of strictly nearly Kähler 6-manifolds are known to exist.

\subsection{Examples of nearly Kähler manifolds}

Just as the conical singularities of parallel $G_{2}$-manifolds correspond to nearly Kähler manifolds, the conical singularities of $\operatorname{Spin}(7)$-manifolds correspond to the so-called "nearly parallel" $G_{2}$-manifolds (see Ivanov [17]). A $G_{2}$-manifold $(M, \omega)$ is called nearly parallel if $d \omega=c * \omega$, where $c$ is some constant. The analogy between nearly Kähler 6-manifolds and nearly parallel $G_{2}$-manifolds is almost perfect. These 
manifolds admit a connection with totally antisymmetric torsion and have weak holonomy SU(3) and $G_{2}$ respectively. Hitchin [16] realized nearly Kähler 6-manifolds and nearly parallel $G_{2}$-manifolds as extrema of a certain functional, called the Hitchin functional by physicists.

However, examples of nearly parallel $G_{2}$-manifolds are found in profusion (every 3-Sasakian manifold is nearly parallel $G_{2}$ ), and compact nearly Kähler manifolds are rare.

Only 4 compact examples are known (see the list below), all of them homogeneous. Butruille [6] showed that any homogeneous nearly Kähler 6-manifold belongs to this list.

(1) The 6-dimensional sphere $S^{6}$. The almost complex structure on $S^{6}$ is reconstructed from the octonion action, and the metric is standard.

(2) $S^{3} \times S^{3}$, with the complex structure mapping $\xi_{i}$ to $\xi_{i}^{\prime}, \xi_{i}^{\prime}$ to $-\xi_{i}$, where $\xi_{i}, \xi_{i}^{\prime}, i=1,2,3$ is a basis of left invariant 1 -forms on the first and the second component.

(3) Given a self-dual Einstein Riemannian 4-manifold $M$ with positive Einstein constant, one defines its twistor space $\operatorname{Tw}(M)$ as a total space of a bundle of unit spheres in $\Lambda_{-}^{2}(M)$ of anti-self-dual 2 -forms. Then $\operatorname{Tw}(M)$ has a natural Kähler-Einstein structure $\left(I_{+}, g\right)$, obtained by interpreting unit vectors in $\Lambda_{-}^{2}(M)$ as complex structure operators on $T M$. Changing the sign of $I_{+}$ on $T M$, we obtain an almost complex structure $I_{-}$which is also compatible with the metric $g$; see Eels and Salamon [8]. A straightforward computation insures that $\left(\operatorname{Tw}(M), I_{-}, g\right)$ is nearly Kähler; see Muškarov [25].

As Hitchin proved, there are only two compact self-dual Einstein 4-manifolds: $S^{4}$ and $\mathbb{C} P^{2}$. The corresponding twistor spaces are $\mathbb{C} P^{3}$ and the flag space $F(1,2)$. The almost complex structure operator $I_{-}$induces a nearly Kähler structure on these two symmetric spaces.

\subsection{Nearly Kähler manifolds in geometry and physics}

In [31] we showed that, unless a nearly Kähler manifold $M$ is locally isometric to a 6-sphere, the almost complex structure on $M$ is uniquely determined by the metric. Friedrich [9] proved this result for $S^{6}$ as well. Also in [31] it was shown that the metric on $M$ is uniquely determined by the almost complex structure.

Denote by $C(M)$ the Riemannian cone of $(M, g)$. By definition, the Riemannian cone is a product $\mathbb{R}^{>0} \times M$, equipped with a metric $t^{2} g+d t^{2}$, where $t$ is a unit parameter of $\mathbb{R}^{>0}$. 
The definition of nearly Kähler manifolds can be reformulated in terms of Riemannian geometry, as follows.

Recall that a spinor $\psi$ is called a Killing spinor if $\nabla_{X} \psi=\lambda X \cdot \psi$ for all vector fields $X \in T M$ and a fixed, nonzero real constant $\lambda$. Any manifold which admits a Killing spinor is Einstein [5]. The following proposition was proven by C Bär.

Proposition 1.3 [4] Let $(M, g)$ be a Riemannian 6-manifold. Then $M$ admits a strictly nearly Kähler almost complex structure if and only if one of the following equivalent conditions holds.

(i) $M$ admits a real Killing spinor.

(ii) The Riemannian cone $C(M)$ has holonomy $G_{2}$.

For an in-depth study of Killing spinors, with applications to 6-dimensional geometry, see Baum et al [5]. From (i) it is apparent that a nearly Kähler manifold is Einstein; indeed, only Einstein manifolds can admit Killing spinors.

Nearly Kähler manifolds appear as the end result of several important classificationtype problems - in the classification of manifolds admitting a Killing spinor, in the classification of conical singularities of $G_{2}$-manifolds, in the classification of manifolds admitting a connection with totally antisymmetric and parallel torsion (see Cleyton and Swann [7]), and so on. These manifolds are even more important in physics, being solutions of type II B string theory; see Friedrich and Ivanov [10]. In that sense, nearly Kähler manifolds are just as important as the usual Calabi-Yau threefolds.

The conical singularities of $G_{2}$-manifolds and the resulting nearly Kähler geometries also known to have applications in physics, giving supergravity solutions which are a product an anti-de Sitter space with an Einstein space (see Acharya et al [1]. More recently, the conical singularities of $G_{2}$-manifolds arising from nearly Kähler geometry were used to obtain string models with chiral matter fields by Atiyah and Witten [3] and Acharya and Witten [2].

\subsection{Local structure of nearly Kähler 6-manifolds}

Let $(M, I, \omega, \Omega)$ be a nearly Kähler manifold. Since $d \omega=3 \lambda \operatorname{Re} \Omega$, the complex structure on $M$ is nonintegrable; indeed, a differential of the $(1,1)$-form $\omega$ lies in $\Lambda^{3,0}(M) \oplus \Lambda^{0,3}(M)$, and this is impossible if $(M, I)$ is integrable.

To fix the notation, we recall some well-known results and calculations relating the de Rham differential and the Nijenhuis tensor. 
An obstruction to integrability of an almost complex structure is given by the Nijenhuis tensor

$$
N^{*}: T^{1,0}(M) \otimes T^{1,0}(M) \longrightarrow T^{0,1}(M),
$$

mapping a pair of $(1,0)$-vector fields to the $(0,1)$-part of their commutator. For our purposes, it is more convenient to deal with its dual, which we denote by the same letter:

$$
N: \Lambda^{0,1}(M) \longrightarrow \Lambda^{2,0}(M) .
$$

From Cartan's formula it is apparent that $N$ is equal to the $(2,-1)$-part of the de Rham differential. On the other hand, $N$ can be expressed through $\nabla I$, in the usual way:

$$
N^{*}(X, Y)=\left(\nabla_{X} I\right) Y-\left(\nabla_{Y} I\right) X,
$$

where $X, Y$ are $(1,0)$-vector fields. On a nearly Kähler manifold, $\nabla(I)$ can be expressed through the 3 -form $d \omega=\nabla \omega$. This gives the following relations (see Kobayashi and Nomizu [22]):

$$
N^{*}(X, Y)=d \omega(X, Y, \cdot)^{\#},
$$

where $d \omega(X, Y, \cdot)^{\#}$ is a vector field dual to the 1 -form $d \omega(X, Y, \cdot)$. Since $d \omega=$ $3 \lambda \operatorname{Re} \Omega$, the relation (1-3) allows one to express $N$ through $\Omega$ and $\omega$.

Let $\xi_{1}, \xi_{2}, \xi_{3} \in \Lambda^{1,0}(M)$ be an orthonormal coframe, such that $\Omega=\xi_{1} \wedge \xi_{2} \wedge \xi_{3}$. Then (1-3) gives

$$
N\left(\bar{\xi}_{1}\right)=\lambda \xi_{2} \wedge \xi_{3}, \quad N\left(\bar{\xi}_{2}\right)=-\lambda \xi_{1} \wedge \xi_{3}, \quad N\left(\bar{\xi}_{3}\right)=\lambda \xi_{1} \wedge \xi_{2} .
$$

This calculation is well known; it is explained in more detail in our paper [31].

\subsection{Hodge decomposition of the de Rham differential and intrinsic Lapla- cians}

The results of this paper can be summarized as follows. Let

$$
d=d^{2,-1}+d^{1,0}+d^{0,1}+d^{-1,2}
$$

be the Hodge decomposition of de Rham differential (Section 3.1). We use the following notation: $d^{2,-1}=: N, d^{-1,2}=: \bar{N}, d^{1,0}=: \partial, d^{0,1}=: \bar{\partial}$.

The usual Kähler identities have a form "a commutator of some Hodge component of de Rham differential with the Hodge operator $\Lambda$ is proportional to a Hermitian adjoint of some other Hodge component of de Rham differential". We prove that a similar set of identities is valid on nearly Kähler manifolds (Theorem 3.1, Proposition 5.1). 
These identities are used to study various intrinsic Laplacians on $M$. We show that the difference

$$
\Delta_{\partial}-\Delta_{\bar{\partial}}=R
$$

is a scalar operator, acting on $(p, q)$-forms as $\lambda^{2}(p-q)(3-p-q)$ (see Corollary 3.3). For the de Rham Laplacian $\Delta_{d}=d d^{*}+d^{*} d$, the following formula holds:

$$
\Delta_{d}=\Delta_{\partial-\bar{\partial}}+\Delta_{N}+\Delta_{\bar{N}}
$$

(see (5-6)). This formula is used to study the harmonic forms on $M$ when $M$ is compact. We show that $\eta$ is harmonic if and only if all Hodge components of $d$ and $d^{*}$ vanish on $\eta$ (Theorem 6.2). This implies that the harmonic forms on $M$ admit a Hodge decomposition:

$$
\mathcal{H}^{*}(M)=\bigoplus \mathcal{H}^{p, q}(M) .
$$

Using (1-5), we obtain that $\mathcal{H}^{p, q}(M)=0$ unless $p=q$ or $(q=2, p=1)$ or $(q=1, p=2)$. We also prove that all harmonic forms $\eta \in \mathcal{H}^{p, q}(M)$, for $(q=2, p=1)$ or $(q=1, p=2)$ or $p=q=2$ are coprimitive, that is, satisfy $\eta \wedge \omega=0$, where $\omega$ is the Hermitian form (Remark 6.4).

\section{Algebraic differential operators on the de Rham algebra}

The following section is purely algebraic. We reproduce some elementary results about algebraic differential operators on graded commutative algebras. There results are later on used to study the de Rham superalgebra.

\subsection{Algebraic differential operators: basic properties}

Let $A^{*}:=\bigoplus^{i} A_{i}$ be a graded commutative ring with unit. For $a \in A_{i}$, we denote by $L_{a}$ the operator of multiplication by $a: L_{a}(\eta)=a \eta$. A supercommutator of two graded endomorphisms $x, y \in \operatorname{End}\left(A^{*}\right)$ is denoted by

$$
\{x, y\}:=x y-(-1)^{\tilde{x} \tilde{y}} y x,
$$

where $\tilde{x}$ denotes the parity of $x$.

Speaking of elements of graded spaces further on in this section, we shall always mean pure elements, that is, elements of pure even or pure odd degree. The parity $\tilde{x}$ is always defined as 1 on odd elements, and 0 on even elements.

Vectors of pure even degree are called even, and vectors of pure odd degree are called odd. 
A supercommutator of two even endomorphisms, or an odd and an even endomorphism is equal to their commutator. We shall sometimes use the usual bracket notation $[\cdot, \cdot]$ in this case.

Definition 2.1 The space $D^{i}\left(A^{*}\right) \subset \operatorname{End}\left(A^{*}\right)$ of algebraic differential operators of algebraic order $i$ is a graded subspace of $\operatorname{End}\left(A^{*}\right)$, which is defined inductively as follows.

(i) $D^{0}(A)$ is a space of $A^{*}$-linear endomorphisms of $A^{*}$, that is, $D^{0}\left(A^{*}\right) \cong A^{*}$.

(ii) $D^{n+1}\left(A^{*}\right)$ is defined as a graded subspace of $\operatorname{End}\left(A^{*}\right)$ consisting of all endomorphisms $\rho \in \operatorname{End}\left(A^{*}\right)$ (even or odd) which satisfy $\left\{L_{a}, \rho\right\} \in D^{n}\left(A^{*}\right)$, for all $a \in A$.

This notion was defined by Grothendieck. Using induction, it is easy to check that $D^{*}\left(A^{*}\right)=\bigcup D^{i}\left(A^{*}\right)$ is a filtered algebra:

$$
\begin{gathered}
D^{i}\left(A^{*}\right) \cdot D^{j}\left(A^{*}\right) \subset D^{i+j}\left(A^{*}\right), \\
\left\{D^{i}\left(A^{*}\right), D^{j}\left(A^{*}\right)\right\} \subset D^{i+j-1}\left(A^{*}\right) .
\end{gathered}
$$

Definition 2.2 Let $\delta: A^{*} \longrightarrow A^{*}$ be an even or odd endomorphism. We say that $\delta$ is a derivation if

$$
\delta(a b)=\delta(a) b+(-1)^{\tilde{a} \tilde{\delta}} a \delta(b),
$$

for any $a, b \in A^{*}$.

Clearly, all derivations of $A^{*}$ are first order algebraic differential operators and vanish on the unit of $A^{*}$. The converse is also true: if $D \subset D^{1}\left(A^{*}\right)$ is a first order differential operator, $D(1)=0$, then $D$ is a derivation, as the following claim implies.

Claim 2.3 Let $D \in D^{1}\left(A^{*}\right)$ be a first order differential operator. Then

$$
D-L_{D(1)}
$$

is a derivation of $A$.

Proof It suffices to prove Claim 2.3 assuming that $D(1)=0$. Let $a, b \in A$ be even or odd elements. Since $\left\{D, L_{a}\right\}$ is $A^{*}$-linear, we have

$$
\begin{aligned}
D(a b)-(-1)^{\tilde{a} \widetilde{D}} a D(b) & =\left\{D, L_{a}\right\}(b)=\left\{D, L_{a}\right\}(1) b \\
& =D(a) b+(-1)^{\tilde{a} \tilde{D}} a D(1)=D(a) b .
\end{aligned}
$$

Remark 2.4 From Claim 2.3, it is clear that a first order differential operator on $A$ is determined by the values taken on 1 and any set of multiplicative generators of $A$. 
The following claim is also clear.

Claim 2.5 Let $D \in \operatorname{End}\left(A^{*}\right)$ be an endomorphism of $A^{*}$, and $V$ a set of generators of $A^{*}$. Assume that for any $v \in V$, we have $\left\{L_{v}, D\right\} \in D^{i}\left(A^{*}\right)$. Then $D$ is an $(i+1)$-st order algebraic differential operator on $A$.

\subsection{Algebraic differential operators on $\Lambda^{*}(M)$}

Let $M$ be a smooth manifold, and $\Lambda^{*}(M)$ its de Rham algebra. It is easily seen that the differential operators (in the usual sense) and the algebraic differential operators on $\Lambda^{*}(M)$ coincide. However, the "algebraic order" of differential operators in the sense of Grothendieck's definition and in the sense of the usual definition are different. For instance, a contraction with a vector field is $C^{\infty}(M)$-linear, hence it has order 0 in the usual sense. However, the contraction with a vector field has algebraic order one in the sense of Definition 2.1. Further on, we always use the term "order" in the sense of "algebraic order", and not in the conventional sense.

From now till the end of this Appendix, the manifold $M$ is always assumed to be Riemannian.

Claim 2.6 Let $M$ be a Riemannian manifold, and $\eta \in \Lambda^{1}(M)$ a 1-form. Denote by $\Lambda_{\eta}$ the metric adjoint to $L_{\eta}, \Lambda_{\eta}=-* L_{\eta} *$. Then $\Lambda_{\eta}$ is a first order differential operator.

Proof Clearly, $\Lambda_{\eta}$ is a contraction with a vector field $\eta^{\sharp}$ dual to $\eta$. Then Claim 2.6 is clear, because a contraction with a vector field is clearly a derivation.

This claim is a special case of the following proposition, which is proved independently.

Proposition 2.7 Let $M$ be a Riemannian manifold, and $\eta \in \Lambda^{n}(M)$ an n-form. Denote by $\Lambda_{\eta}$ the metric adjoint to $L_{a}, \Lambda_{\eta}=(-1)^{\widetilde{\eta}} * L_{\eta} *$. Then $\Lambda_{\eta}$ is a differential operator of algebraic order $n$.

Proof We use the induction on $n$. For $n=0$ everything is clear. As Claim 2.5 implies, to prove that $\Lambda_{\eta} \in D^{n}\left(\Lambda^{*}(M)\right)$, we need to show that

$$
\left\{\Lambda_{\eta}, L_{a}\right\} \in D^{n-1}\left(\Lambda^{*}(M)\right),
$$

for any $a \in \Lambda^{0}(M), \Lambda^{1}(M)$. For $a \in \Lambda^{0}(M),(2-3)$ is clear, because $\Lambda_{\eta}$ is $C^{\infty}(M)$ linear, hence $\left\{\Lambda_{\eta}, L_{a}\right\}=0$. For $a \in \Lambda^{1}(M)$, it is easy to check that

$$
\left\{\Lambda_{\eta}, L_{a}\right\}=\Lambda_{\eta\lrcorner a^{\sharp}}
$$

where $a^{\sharp}$ is the dual vector field, and $\lrcorner$ a contraction. The induction statement immediately gives (2-3). 


\subsection{An algebraic differential operator and its adjoint}

The main result of this section is the following proposition.

Proposition 2.8 Let $(M, g)$ be a Riemannian manifold, and

$$
D: \Lambda^{*}(M) \longrightarrow \Lambda^{*+1}(M)
$$

a first order algebraic differential operator. Denote by $D^{*}$ its metric adjoint, $D^{*}=-* D *$. Then $D^{*}$ is a second order algebraic differential operator.

Proof Step 1 As follows from Claim 2.5, it suffices to check that

$$
\left\{\left\{D^{*}, L_{a}\right\}, L_{b}\right\} \text { is } \Lambda^{*}(M) \text {-linear, }
$$

for all $a, b \in \Lambda^{0}(M), \Lambda^{1}(M)$.

Step 2 We use the following lemma.

Lemma 2.9 Let $D_{1}: \Lambda^{*}(M) \longrightarrow \Lambda^{*-1}(M)$ be a first order algebraic differential operator decreasing the degree by 1 . Then $D_{1}=0$.

Proof This follows from Remark 2.4.

Step 3 Letting $D_{1}:=\left\{\left\{D, \Lambda_{a}\right\}, \Lambda_{b}\right\}$, clearly,

$$
\left\{\left\{D^{*}, L_{a}\right\}, L_{b}\right\}^{*}=D_{1}^{*} \text {. }
$$

From Claim 2.6 and (2-2), we find that $D_{1}$ is an algebraic differential operator of algebraic order 1 (being a commutator of several first order operators). When $a, b \in$ $\Lambda^{1}(M), D_{1}$ decreases the degree of a form by 1 . By Claim $2.3, D_{1}$ is a derivation. Clearly, a derivation which vanishes on $\Lambda^{0}(M)$ is $C^{\infty}(M)$-linear. This shows that $D_{1}$ is $C^{\infty}(M)$-linear.

By Lemma 2.9, the commutator of $D_{1}$ with $\Lambda_{c}$ vanishes, for all $c \in \Lambda^{1}(M)$ :

$$
\left\{D_{1}, \Lambda_{c}\right\}=0 .
$$

The operator $D_{1}^{*}=\left\{\left\{D^{*}, L_{a}\right\}, L_{b}\right\}$ is $C^{\infty}(M)$-linear (being adjoint to $D_{1}$ ), and commutes with all $L_{a}$, as follows from (2-5). Therefore, $D_{1}^{*}$ is $\Lambda^{*}(M)$-linear. This proves (2-4) for $a, b \in \Lambda^{1}(M)$.

Step 4 Clearly, $L_{a}=\Lambda_{a}$ when $a \in \Lambda^{0}(M)$. Then

$$
\left\{\left\{D^{*}, L_{a}\right\}, L_{b}\right\}=\left\{\left\{D^{*}, \Lambda_{a}\right\}, \Lambda_{b}\right\}=\left\{\left\{D, L_{a}\right\}, L_{b}\right\}^{*}=0,
$$


because $D$ is a first order algebraic differential operator. This proves (2-4) for $a, b \in \Lambda^{0}(M)$.

Step 5 Since the algebra $\Lambda^{*}(M)$ is graded commutative, $\left\{L_{a}, L_{b}\right\}=0$ for all $a, b \in \Lambda^{*}(M)$. Using the graded Jacobi identity, we find that

$$
\left\{\left\{D^{*}, L_{a}\right\}, L_{b}\right\}=(-1)^{\tilde{a} \tilde{b}}\left\{\left\{D^{*}, L_{b}\right\}, L_{a}\right\},
$$

for all $a, b$. In Steps 3 and 4 we proved (2-4) for $a, b \in \Lambda^{1}(M), a, b \in \Lambda^{0}(M)$. By (2-7), to prove Proposition 2.8 it remains to show that $\left\{\left\{D^{*}, L_{a}\right\}, L_{b}\right\}$ is $\Lambda^{*}(M)$ linear for $a \in \Lambda^{0}(M), b \in \Lambda^{1}(M)$.

Step 6 In this case,

$$
\left\{D^{*}, L_{a}\right\}=\left\{D^{*}, \Lambda_{a}\right\}=\left\{D, L_{a}\right\}^{*}=L_{D(a)}^{*}=\Lambda_{D(a)} .
$$

Then

$$
\left\{\left\{D^{*}, L_{a}\right\}, L_{b}\right\}=\left\{\Lambda_{D(a)}, L_{b}\right\}=g(D(a), b),
$$

because $\Lambda_{D(a)}$ is a contraction with the dual vector field $D(a)^{\#}$. We proved that $\left\{\left\{D^{*}, L_{a}\right\}, L_{b}\right\}$ is a scalar function, hence it is $\Lambda^{*}(M)$-linear. Proposition 2.8 is proved.

\section{Kähler identities on nearly Kähler manifolds}

\subsection{The operators $\partial, \bar{\partial}$ on almost complex manifolds}

Let $(M, I)$ be an almost complex manifold, and $d: \Lambda^{i}(M) \longrightarrow \Lambda^{i+1}(M)$ the de Rham differential. The Hodge decomposition gives

$$
d=\bigoplus_{i+j=1} d^{i, j}, \quad d^{i, j}: \Lambda^{p, q}(M) \longrightarrow \Lambda^{p+i, q+j}(M)
$$

Using the Leibniz identity, we find that the differential and all its Hodge components are determined by the values taken on all vectors in the spaces $\Lambda^{0}(M), \Lambda^{1}(M)$, generating the de Rham algebra. On $\Lambda^{0}(M)$, only $d^{1,0}, d^{0,1}$ can be nonzero, and on $\Lambda^{1}(M)$ only $d^{2,-1}, d^{1,0}, d^{0,1}, d^{-1,2}$ can be nonzero. Therefore, only 4 components of (3-1) can be possibly nonzero:

$$
d=d^{2,-1}+d^{1,0}+d^{0,1}+d^{-1,2} .
$$

Since $N:=d^{2,-1}, \bar{N}:=d^{-1,2}$ vanish on $\Lambda^{0}(M)$, these components are $C^{\infty}(M)-$ linear. In fact,

$$
N: \Lambda^{0,1}(M) \longrightarrow \Lambda^{2,0}(M)
$$


is the Nijenhuis tensor (1-2) of $(M, I)$, extended to an operator on $\Lambda^{*}(M)$ by the Leibniz rule. We denote $d^{1,0}$ as $\partial: \Lambda^{p, q}(M) \longrightarrow \Lambda^{p+1, q}(M)$, and $d^{0,1}$ as $\bar{\partial}: \Lambda^{p, q}(M) \longrightarrow \Lambda^{p, q+1}(M)$. Decomposing $d^{2}=0$, we find

$$
\begin{array}{r}
N^{2}+\{N, \partial\}+\left(\{\bar{\partial}, N\}+\partial^{2}\right)+(\{N, \bar{N}\}+\{\partial, \bar{\partial}\})+\left(\{\partial, \bar{N}\}+\bar{\partial}^{2}\right)+\{\bar{N}, \bar{\partial}\}+\bar{N}^{2} \\
=d^{2}=0,
\end{array}
$$

where $\{\cdot, \cdot\}$ denotes the supercommutator. The terms in brackets in (3-2) are different Hodge components of $d^{2}$, and since $d^{2}=0$, they all vanish:

$$
\begin{aligned}
N^{2} & =\{N, \partial\}=\{\bar{\partial}, N\}+\partial^{2} \\
& =\{N, \bar{N}\}+\{\partial, \bar{\partial}\}=\{\partial, \bar{N}\}+\bar{\partial}^{2} \\
& =\{\bar{N}, \bar{\partial}\}=\bar{N}^{2}=0 .
\end{aligned}
$$

However, the operators $\partial^{2}$ and $\bar{\partial}^{2}$ can be nonzero.

The following almost complex version of the Kähler identities is quite useful further on in our study.

Theorem 3.1 Let $(M, I)$ be an almost complex Hermitian manifold, $\omega \in \Lambda^{1,1}(M)$ a Hermitian form, and $\Lambda_{\omega}: \Lambda^{i}(M) \longrightarrow \Lambda^{i-2}(M)$ a Hermitian adjoint to $L_{\omega}(\eta)=$ $\omega \wedge \eta$. Consider the operators $\partial, \bar{\partial}: \Lambda^{i}(M) \longrightarrow \Lambda^{i+1}(M)$ constructed above, and let $\partial^{*}, \bar{\partial}^{*}: \Lambda^{i}(M) \longrightarrow \Lambda^{i-1}(M)$ be the corresponding Hermitian adjoint operators. Assume that $d \omega \in \Lambda^{3,0}(M) \oplus \Lambda^{0,3}(M)$, that is, $\partial \omega=\bar{\partial} \omega=0$. Then

$$
\begin{array}{rlrl}
{\left[\Lambda_{\omega}, \partial\right]} & =\sqrt{-1} \bar{\partial}^{*}, & {\left[\Lambda_{\omega}, \bar{\partial}\right]} & =-\sqrt{-1} \partial^{*}, \\
{\left[L_{\omega}, \partial^{*}\right]} & =\sqrt{-1} \bar{\partial}, & {\left[\Lambda_{\omega}, \partial\right]=\sqrt{-1} \bar{\partial}^{*} .}
\end{array}
$$

Proof To prove Theorem 3.1, we use essentially the same argument as used in the proof of the conventional Kähler identities in the situation when a coordinate approach does not work; see eg our proof of Kähler identities in HKT-geometry, obtained in [29], and our proof of the Kähler identities in locally conformally hyperkähler geometry, obtained in [30].

Equations (3-4) and (3-5) are Hermitian adjoint, hence equivalent. The two equations (3-5) are complex conjugate, hence they are also equivalent. To prove Theorem 3.1 it is sufficient to prove only one of these equations, say

$$
\left[L_{\omega}, \partial^{*}\right]=\sqrt{-1} \bar{\partial} .
$$

The proof of such a relation follows a general template, which is given in Section 2. There is an algebraic notion of differential operators on a graded commutative algebra, 
due to Grothendieck (Definition 2.1). In this sense, the operators $\partial^{*}, \bar{\partial}^{*}$ are second order algebraic differential operators on $\Lambda^{*}(M)$ (see Proposition 2.8), and $L_{\omega}$ is $\Lambda^{*}(M)$-linear. Then $\left[L_{\omega}, \partial^{*}\right]$ (being a commutator of 0 -th and $2-$ nd order algebraic differential operators on $\left.\Lambda^{*}(M)\right)$ is a first order algebraic differential operator. To prove a relation between first order algebraic differential operators on an algebra, such as (3-6), it suffices to check it on any set of generators of this algebra 2.4. To prove Theorem 3.1 it remains to show that (3-6) holds on some set of generators, eg 1-forms and 0 -forms.

Given a function $f \in C^{\infty}(M)$, we have

$$
\left[L_{\omega}, \partial^{*}\right] f=-\partial^{*}(f \omega) .
$$

However, $\partial^{*}=-* \partial *$ and $*(f \omega)=\bar{f} \omega^{n-1}$, where $n=\operatorname{dim}_{\mathbb{C}} M$. Then

$$
\left[L_{\omega}, \partial^{*}\right] f=*\left(\partial \bar{f} \wedge \omega^{n-1}\right)=\sqrt{-1} \bar{\partial} f
$$

because for any $(1,0)-$ form $\eta$ we have $*\left(\eta \wedge \omega^{n-1}\right)=\sqrt{-1} \bar{\eta}$, and $\overline{\partial \bar{f}}=\bar{\partial} f$.

It is easy to check that $\partial^{*}$-closed 1 -forms generate the bundle of all 1 -forms over $C^{\infty}(M)$. Indeed, on 2 -forms we have $\left(\partial^{*}\right)^{2}=0$, and therefore, all $\partial^{*}$-exact $1-$ forms are $\partial^{*}$-closed. A local calculation implies that $\partial^{*}\left(\Lambda^{2}(M)\right)$ generates $\Lambda^{1}(M)$ over $C^{\infty}(M)$.

Consider a 1 -form $\eta \in \Lambda^{1}(M)$. To prove Theorem 3.1 it remains to show that $\left[L_{\omega}, \partial^{*}\right](\eta)=\sqrt{-1} \bar{\partial} \eta$. Since $\partial^{*}$-closed 1 -forms generate $\Lambda^{*}(M)$, we may assume that $\eta$ is $\partial^{*}$-closed. Then

$$
\begin{aligned}
(\eta) & =-\partial^{*} L_{\omega} \eta=* \partial *(\omega \wedge \eta) \\
& =* \partial\left(\omega^{n-2} \wedge I(\bar{\eta})\right)=*\left(\omega^{n-2} \wedge \partial(I \bar{\eta})\right) .
\end{aligned}
$$

Since $\eta$ is $\partial^{*}$-closed, we have $\omega^{n-1} \wedge \partial(I \bar{\eta})=0$, and therefore the form $\omega^{n-2} \wedge \partial \eta$ is coprimitive (satisfies $\left.\left(\omega^{n-2} \wedge \partial \eta\right) \wedge \omega=0\right)$. Given a coprimitive $(2 n-2)$-form $\alpha=\kappa \wedge \omega^{n-2}$, the form $* \alpha$ can be written down explicitly in terms of $\kappa: * \alpha=-I(\bar{\kappa})$. Then

$$
*\left(\omega^{n-2} \wedge \partial I(\bar{\eta})\right)=-\overline{I \partial I \bar{\eta}}=\sqrt{-1} \bar{\partial} \eta .
$$

Comparing this with (3-8), we find that

$$
\left[L_{\omega}, \partial^{*}\right](\eta)=\sqrt{-1} \bar{\partial} \eta
$$

This proves Theorem 3.1. 


\subsection{The Nijenhuis operator squared}

Later on, we shall need the following useful identity.

Proposition 3.2 Let $(M, I, \omega, \Omega)$ be a nearly Kähler 6-manifold, $d \omega=\lambda \operatorname{Re} \Omega$, and $C:=N+\bar{N}=d^{2,-1}+d^{-1,2}$ the $(2,-1) \oplus(-1,2)-$ part of the de Rham differential. Then the following $C^{\infty}(M)$-linear maps

$$
\Lambda^{p, q}(M) \longrightarrow \Lambda^{p+1, q+1}(M)
$$

are equal:

(i) $C^{2}$,

(ii) $-\{\partial, \bar{\partial}\}$,

(iii) the scalar operator $\sqrt{-1} \lambda^{2}(p-q) L_{\omega}$, mapping $\eta \in \Lambda^{p, q}(M)$ to

$$
\sqrt{-1} \lambda^{2}(p-q) \eta \wedge \omega \text {. }
$$

Proof The equivalence $C^{2}=-\{\partial, \bar{\partial}\}$ is clear, because the $(1,1)-$ part of $d^{2}$ is equal to $C^{2}+\{\partial, \bar{\partial}\}$, and $d^{2}=0$ (see (3-3)). To prove

$$
C^{2}=\sqrt{-1} \lambda^{2}(p-q) L_{\omega},
$$

we notice that both sides of (3-9) are differentiations ( $C^{2}$ being a supercommutator of an odd differentiation with itself). Therefore it suffices to check (3-9) only on the generators of $\Lambda^{*}(M)$, eg on $\Lambda^{0}(M)$ and $\Lambda^{1}(M)$. On $\Lambda^{0}(M)$, both $C$ and $(p-q)$ vanish, hence (3-9) is clear. Let us check (3-9) on $\Lambda^{1,0}(M)$ (a proof of (3-9) on $\Lambda^{0,1}(M)$ is obtained in the same fashion). Choose an orthonormal frame $\xi_{1}, \xi_{2}, \xi_{3} \in \Lambda^{1,0}(M)$, in such a way that

$$
\omega=-\sqrt{-1}\left(\xi_{1} \wedge \bar{\xi}_{1}+\xi_{2} \wedge \bar{\xi}_{2}+\xi_{3} \wedge \bar{\xi}_{3}\right), \quad \Omega=\xi_{1} \wedge \xi_{2} \wedge \xi_{3} .
$$

Let $\eta$ be a $(0,1)$-form, say, $\eta=\bar{\xi}_{1}$ (this assumption is not restrictive, because both sides of (3-9) are manifestly $C^{\infty}(M)$-linear). Then $N(\eta)=\lambda \xi_{2} \wedge \xi_{3}$, as (1-4) implies. Similarly, the Leibniz rule and (1-4) give

$$
\bar{N} N(\eta)=\lambda^{2}\left(\bar{\xi}_{1} \wedge \bar{\xi}_{3} \wedge \xi_{3}+\xi_{1} \wedge \bar{\xi}_{1} \wedge \bar{\xi}_{2}\right)=\sqrt{-1} \lambda^{2} \eta \wedge \omega .
$$

On the other hand,

$$
C^{2}(\eta)=(N+\bar{N})^{2} \eta=\bar{N} N(\eta)
$$

because $\bar{N} \eta$ vanishes, being a $(-1,3)$-form, and $N^{2}=\bar{N}^{2}=0$ as (3-2) implies. Combining (3-10) and (3-11), we obtain (3-9). Proposition 3.2 is proved. 
Corollary 3.3 Let $(M, I, \omega, \Omega)$ be a nearly Kähler 6-manifold, $d \omega=\lambda \operatorname{Re} \Omega$, and $\partial, \bar{\partial}$ the $(1,0)$ - and $(0,1)$-parts of de Rham differential. Consider the corresponding Laplacians:

$$
\Delta_{\partial}:=\partial \partial^{*}+\partial^{*} \partial, \quad \Delta \bar{\partial}:=\overline{\partial \partial}^{*}+\bar{\partial}^{*} \bar{\partial} .
$$

Then $\Delta_{\partial}-\Delta_{\bar{\partial}}=R$, where $R$ is a scalar operator acting on $(p, q)$-forms as a multiplication by $\lambda^{2}(3-p-q)(p-q)$.

Proof As Proposition 3.2 implies, $\{\partial, \bar{\partial}\}=-\sqrt{-1}(p-q) \lambda^{2} L_{\omega}$. It is well known that $H:=\left[L_{\omega}, \Lambda_{\omega}\right]$ acts on $(p, q)$-forms as a multiplication by $(3-p-q)$ (see eg Griffiths and Harris [15]). Then

$$
\left\{\Lambda_{\omega},\{\partial, \bar{\partial}\}\right\}=\sqrt{-1} R .
$$

Applying the graded Jacobi identity and Theorem 3.1 to (3-12), we obtain

$$
\sqrt{-1} R=\left\{\Lambda_{\omega},\{\partial, \bar{\partial}\}\right\}=\left\{\left\{\Lambda_{\omega}, \partial\right\}, \bar{\partial}\right\}+\left\{\partial,\left\{\Lambda_{\omega}, \bar{\partial}\right\}\right\}=\sqrt{-1} \Delta_{\partial}-\sqrt{-1} \Delta_{\bar{\partial}} .
$$

This proves Corollary 3.3.

\section{The de Rham Laplacian via $\Delta_{\partial}, \Delta_{\bar{\partial}}, \Delta_{\partial-\bar{\partial}}$}

\subsection{An expression for $\Delta_{d}$}

Let $M$ be a nearly Kähler 6-manifold, $d=N+\partial+\bar{\partial}+\bar{N}$ the Hodge decomposition of the de Rham differential, and $\Delta_{\partial}, \Delta_{\bar{\partial}}$ the Laplacians defined above, $\Delta_{\partial}:=\left\{\partial, \partial^{*}\right\}$, $\Delta \bar{\partial}:=\left\{\bar{\partial}, \bar{\partial}^{*}\right\}$. Denote the usual (Hodge-de Rham) Laplacian by $\Delta_{d}=\left\{d, d^{*}\right\}$. In addition to Corollary 3.3, the following relation between the Laplacians can be obtained.

Theorem 4.1 Let $M$ be a nearly Kähler 6-manifold, and $\Delta_{\partial}, \Delta_{\bar{\partial}}, \Delta_{d}$ the Laplacians considered above. Then

$$
\Delta_{d}=\Delta_{\partial}+\Delta_{\bar{\partial}}+\Delta_{N+\bar{N}}-\left\{\partial, \bar{\partial}^{*}\right\}-\left\{\bar{\partial}, \partial^{*}\right\},
$$

where $\Delta_{N+\bar{N}}$ is defined as a supercommutator of the $C^{\infty}$-linear operator $C:=N+\bar{N}$ and its Hermitian adjoint:

$$
\Delta_{N+\bar{N}}:=C C^{*}+C^{*} C
$$

The proof of Theorem 4.1 takes the rest of this section. 


\section{$4.2 N=\lambda\left[L_{\Omega}, \Lambda_{\omega}\right]$}

The following linear-algebraic relation is used further on in the proof of Theorem 4.1.

Claim 4.2 Let $(M, I, \omega, \Omega)$ be a nearly Kähler 6-manifold which satisfies $d \omega=$ $\lambda \operatorname{Re} \Omega, N$ the $(2,-1)$-part of the de Rham differential, and $\Lambda_{\omega}$ the Hodge operator defined above. Then

$$
\lambda\left[L_{\Omega}, \Lambda_{\omega}\right]=N
$$

where $L_{\Omega}(\eta):=\Omega \wedge \eta$.

Proof As in the proof of Theorem 3.1, we consider $L_{\Omega}, \Lambda_{\omega}$ as algebraic differential operators on the graded commutative algebra $\Lambda^{*}(M)$ (see Definition 2.1). Then $L_{\Omega}$ is a 0 -th order operator, and $\Lambda_{\omega}$ a second order operator, as follows from Proposition 2.7. Therefore, the commutator $\left[L_{\Omega}, \Lambda_{\omega}\right]$ is a first order operator. By Remark 2.4 it suffices to check (4-2) on 0 - and 1-forms. This can be done by an explicit calculation, using (1-4).

\subsection{Commutator relations for $N, \bar{N}, \partial, \bar{\partial}$}

Proposition 4.3 Under the assumptions of Theorem 4.1, the following anticommutators vanish:

$$
\left\{N^{*}, \bar{\partial}\right\}=\left\{\bar{N}^{*}, \partial\right\}=\left\{N, \bar{\partial}^{*}\right\}=\left\{\bar{N}, \partial^{*}\right\}=0 .
$$

Moreover, we have

$$
\begin{aligned}
& \left\{\bar{\partial}^{*}, \partial\right\}=-\left\{N, \partial^{*}\right\}=-\left\{\bar{N}^{*}, \bar{\partial}\right\}, \\
& \left\{\partial^{*}, \bar{\partial}\right\}=-\left\{\bar{N}, \bar{\partial}^{*}\right\}=-\left\{N^{*}, \partial^{*}\right\} .
\end{aligned}
$$

Proof Clearly, all relations of (4-3) can be obtained by applying the complex conjugation and taking the Hermitian adjoint of the relation

$$
\left\{N^{*}, \bar{\partial}\right\}=0 \text {. }
$$

Decomposing $d^{2}=0$ onto Hodge components, we obtain $\{N, \partial\}=0$ (see (3-3)). By Claim 4.2, this is equivalent to

$$
\left\{\left\{L_{\Omega}, \Lambda_{\omega}\right\}, \partial\right\}=0 .
$$

Clearly, $\partial \Omega=0$, hence

$$
\left\{L_{\Omega}, \partial\right\}=0 .
$$


Applying the graded Jacobi identity to (4-6) and using (4-7), we obtain

$$
0=\left\{L_{\Omega},\left\{\Lambda_{\omega}, \partial\right\}\right\}=\sqrt{-1}\left\{L_{\Omega}, \bar{\partial}^{*}\right\} .
$$

Acting on (4-8) by $\left\{\Lambda_{\omega}, \cdot\right\}$ and using the graded Jacobi identity, we obtain

$$
0=\left\{\Lambda_{\omega},\left\{L_{\Omega}, \bar{\partial}^{*}\right\}\right\}=-\lambda\left\{N, \bar{\partial}^{*}\right\} .
$$

This proves (4-5) and (4-3).

It remains to prove (4-4). Taking a Hodge component of $d^{2}=0$, we obtain

$$
\frac{1}{2}\{\partial, \partial\}+\{N, \bar{\partial}\}=0
$$

(see (3-3)). Using the same argument as gives (4-7), we find

$$
\begin{aligned}
\{N, \bar{\partial}\}=-\lambda^{-1}\left\{\left\{\Lambda_{\omega}, L_{\Omega}\right\}, \bar{\partial}\right\} & =\lambda^{-1}\left\{\left\{L_{\Omega},\left\{\Lambda_{\omega}, \bar{\partial}\right\}\right\}-\lambda^{-1}\left\{\Lambda_{\omega},\left\{L_{\Omega}, \bar{\partial}\right\}\right\}\right. \\
& =-\sqrt{-1} \lambda^{-1}\left\{L_{\Omega}, \partial^{*}\right\} .
\end{aligned}
$$

Together with (4-9), this gives

$$
\frac{1}{2}\{\partial, \partial\}=\sqrt{-1} \lambda^{-1}\left\{L_{\Omega}, \partial^{*}\right\} .
$$

Acting on (4-10) with $\left\{\Lambda_{\omega}, \cdot\right\}$, we obtain

$$
\sqrt{-1}\left\{\partial, \bar{\partial}^{*}\right\}=\sqrt{-1} \lambda^{-1}\left\{\left\{\Lambda_{\omega}, L_{\Omega}\right\}, \partial^{*}\right\}=-\sqrt{-1}\left\{N, \partial^{*}\right\} .
$$

We obtained the first equation of (4-4):

$$
\left\{\partial, \bar{\partial}^{*}\right\}=-\left\{N, \partial^{*}\right\} .
$$

This proves Proposition 4.3.

\subsection{The Hodge decomposition of the Laplacian}

Now we can finish the proof of Theorem 4.1. Decomposing $d, d^{*}$ onto Hodge components, we obtain

$$
\begin{aligned}
\Delta_{d}= & \left(\left\{N^{*}, \bar{\partial}\right\}+\left\{N, \bar{\partial}^{*}\right\}+\left\{\bar{N}^{*}, \partial\right\}+\left\{\bar{N}, \partial^{*}\right\}\right) \\
& +\left(\left\{\partial^{*}, \bar{\partial}\right\}+\left\{\bar{\partial}^{*}, \partial\right\}+\left\{N, \partial^{*}\right\}+\left\{\bar{N}, \bar{\partial}^{*}\right\}+\left\{N^{*}, \partial\right\}+\left\{\bar{N}^{*}, \bar{\partial}\right\}\right) \\
& +\Delta_{\partial}+\Delta \bar{\partial}+\Delta_{N+\bar{N}}
\end{aligned}
$$

The first term in brackets vanishes by (4-3), and the second term is equal $-\left\{\partial, \bar{\partial}^{*}\right\}-$ $\left\{\bar{\partial}, \partial^{*}\right\}$ as (4-4) implies. This gives

$$
\Delta_{d}=\Delta_{\partial}+\Delta_{\bar{\partial}}+\Delta_{N+\bar{N}}-\left\{\partial, \bar{\partial}^{*}\right\}-\left\{\bar{\partial}, \partial^{*}\right\},
$$

which proves Theorem 4.1. 
The relation (4-13) can be rewritten as the following relation between Laplacians.

Corollary 4.4 Under the assumptions of Theorem 4.1, denote by $\Delta_{\partial-\bar{\partial}}$ the Laplacian $\left\{\partial-\bar{\partial}, \partial^{*}-\bar{\partial}^{*}\right\}$. Then

$$
\Delta_{d}=\Delta_{\partial-\bar{\partial}}+\Delta_{N+\bar{N}}
$$

Proof As (4-13) implies, to prove (4-14) we need to show that

$$
\Delta_{\partial-\bar{\partial}}=\Delta_{\partial}+\Delta_{\bar{\partial}}-\left\{\partial, \bar{\partial}^{*}\right\}-\left\{\bar{\partial}, \partial^{*}\right\} .
$$

This is clear.

\section{Kähler-type identities for $N, \bar{N}$}

Further on, we shall need the following analogue of Kähler relations (Theorem 3.1), but for the $C^{\infty}(M)$-linear "outer" parts of the de Rham differential, $N$ and $\bar{N}$.

Proposition 5.1 Let $(M, I, \omega, \Omega)$ be a nearly Kähler 6-manifold, $N, \bar{N}$ the $(2,-1)$ and $(-1,2)$-parts of the de Rham differential, $N^{*}, \bar{N}^{*}$ their Hermitian adjoints operators, and $\Lambda_{\omega}$ the Hermitian adjoint to $L_{\omega}(\eta):=\omega \wedge \eta$. Then

$$
\begin{aligned}
{\left[\Lambda_{\omega}, N^{*}\right] } & =2 \sqrt{-1} \bar{N}, & {\left[\Lambda_{\omega}, \bar{N}^{*}\right] } & =-2 \sqrt{-1} N, \\
{\left[L_{\omega}, N\right] } & =2 \sqrt{-1} \bar{N}^{*}, & {\left[L_{\omega}, \bar{N}\right] } & =-2 \sqrt{-1} N^{*} .
\end{aligned}
$$

Proof The equalities of (5-1) are obtained one from another by taking complex conjugation and Hermitian adjoint, hence they are equivalent. Therefore, it suffices to prove

$$
\left[L_{\omega}, N^{*}\right]=2 \sqrt{-1} \bar{N} .
$$

The proof of this formula follows the same lines as the proof of Theorem 3.1. Again, both sides of (5-2) are first order algebraic differential operators on the algebra $\Lambda^{*}(M)$, in the sense of Grothendieck (Definition 2.1). Therefore, it suffices to check (5-2) only on 0 -forms and 1-forms (Remark 2.4). On 0-forms, both sides of (5-2) clearly vanish. To finish the proof of (5-2), it remains to check that

$$
N^{*} L_{\omega}(\eta)=2 \sqrt{-1} \bar{N}(\eta),
$$

where $\eta$ is a 1 -form. Since both sides of (5-3) vanish on $(1,0)$-forms, we may also assume that $\eta \in \Lambda^{0,1}(M)$. 
Let $\xi_{1}, \xi_{2}, \xi_{2}$ be an orthonormal frame in $\Lambda^{1,0}(M)$, satisfying

$$
\omega=-\sqrt{-1}\left(\xi_{1} \wedge \bar{\xi}_{1}+\xi_{2} \wedge \bar{\xi}_{2}+\xi_{3} \wedge \bar{\xi}_{3}\right), \quad \Omega=\xi_{1} \wedge \xi_{2} \wedge \xi_{3} .
$$

Since both sides of (5-2) are $C^{\infty}(M)$-linear, we need to prove (5-2) only for $\eta=$ $\bar{\xi}_{1}, \bar{\xi}_{2}, \bar{\xi}_{2}$. Assume for example that $\eta=\bar{\xi}_{1}$. Then

$$
L_{\omega} \eta=-\sqrt{-1} \bar{\xi}_{1} \wedge\left(\xi_{2} \wedge \bar{\xi}_{2}+\xi_{3} \wedge \bar{\xi}_{3}\right) .
$$

Using (1-4), we obtain

$$
N^{*} L_{\omega} \eta=-\sqrt{-1} \xi_{3} \wedge \xi_{2}+\sqrt{-1} \xi_{2} \wedge \xi_{3}=2 \sqrt{-1} \xi_{2} \wedge \xi_{3}=2 \sqrt{-1} \bar{N}(\eta) .
$$

This proves (5-2), completing the proof of Proposition 5.1.

Proposition 5.1 is used in this paper only once, to obtain the following corollary.

Corollary 5.2 Let $M$ be a nearly Kähler 6-manifold, $N, \bar{N}$ the $(2,-1)$ - and $(-1,2)$-parts of the de Rham differential, $N^{*}, \bar{N}^{*}$ their Hermitian adjoint operators, and $\Delta_{N}, \Delta_{\bar{N}}, \Delta_{N+\bar{N}}$ the corresponding Laplacians. Then

$$
\Delta_{N+\bar{N}}=\Delta_{N}+\Delta_{\bar{N}}
$$

Proof Clearly, we have

$$
\Delta_{N+\bar{N}}=\Delta_{N}+\Delta_{\bar{N}}+\left\{N, \bar{N}^{*}\right\}+\left\{\bar{N}, N^{*}\right\} .
$$

Therefore, to prove Corollary 5.2, it suffices to show that

$$
\left\{N, \bar{N}^{*}\right\}=0, \quad\left\{\bar{N}, N^{*}\right\}=0 .
$$

One of these equations is obtained from another by complex conjugation; therefore, they are equivalent. Let us prove, for instance, $\left\{N, \bar{N}^{*}\right\}=0$. As follows from Proposition 5.1,

$$
\left\{N, \bar{N}^{*}\right\}=-\frac{\sqrt{-1}}{2}\left\{N,\left\{\Lambda_{\omega}, N\right\}\right\} .
$$

However, $\{N, N\}=0$ as follows from (3-3). Using the graded Jacobi identity, we obtain

$$
0=\left\{\Lambda_{\omega},\{N, N\}\right\}=\left\{\left\{\Lambda_{\omega}, N\right\}, N\right\}+\left\{N,\left\{\Lambda_{\omega}, N\right\}\right\}=2\left\{N,\left\{\Lambda_{\omega}, N\right\}\right\} .
$$

Therefore, (5-4) implies $\left\{N, \bar{N}^{*}\right\}=0$. This proves Corollary 5.2. 
Remark 5.3 From Corollary 5.2, Corollary 4.4 and Theorem 4.1, we infer that

$$
\begin{aligned}
& \Delta_{d}=\Delta_{\partial}+\Delta_{\bar{\partial}}+\Delta_{N}+\Delta_{\bar{N}}-\left\{\partial^{*}, \bar{\partial}\right\}-\left\{\partial, \bar{\partial}^{*}\right\}, \\
& \Delta_{d}=\Delta_{\partial-\bar{\partial}}+\Delta_{N}+\Delta_{\bar{N}} .
\end{aligned}
$$

\section{Harmonic forms on nearly Kähler manifolds}

\subsection{Harmonic forms and the $\partial-, \bar{\partial}-$ Laplacians}

For harmonic forms on a compact nearly Kähler manifold, the relation (4-1) of Theorem 4.1 can be strengthened significantly.

Theorem 6.1 Let $M$ be a compact nearly Kähler 6-manifold, and $\eta$ a differential form. Then $\eta$ is harmonic if and only if

$\eta \in \operatorname{ker} \Delta_{\partial} \cap \operatorname{ker} \Delta_{\bar{\partial}} \cap \operatorname{ker} \Delta_{N+\bar{N}}$.

Proof The "if" part is clear; indeed, if (6-1) is true, then $\partial \eta=\bar{\partial} \eta=\partial^{*} \eta=\bar{\partial}^{*} \eta=0$, and $\Delta_{d} \eta=0$ by Theorem 4.1 .

As Corollary 4.4 implies,

$$
\Delta_{d} \eta=0 \Longleftrightarrow\left(\Delta_{\partial-\bar{\partial}} \eta=0 \text { and } \Delta_{N+\bar{N}} \eta=0\right) .
$$

Therefore, for any $\Delta_{d}$-harmonic form $\eta$, we have $\Delta_{\partial-\bar{\partial}} \eta=0$, that is, $(\partial-\bar{\partial}) \eta=0$ and $\left(\partial^{*}-\bar{\partial}^{*}\right) \eta=0$. Moreover, since $d \eta=(N+\bar{N}) \eta=0, \partial+\bar{\partial}=d-N-\bar{N}$ vanishes on $\eta$ as well. Subtracting from $(\partial+\bar{\partial}) \eta=0$ the relation $(\partial-\bar{\partial}) \eta=0$, we obtain that $\bar{\partial} \eta=0$. In a similar way one proves the whole set of equations

$$
\partial \eta=\bar{\partial} \eta=\partial^{*} \eta=\bar{\partial}^{*} \eta=0 .
$$

This gives (6-1).

\subsection{The Hodge decomposition on cohomology}

The main result of this paper is an immediate corollary of Theorem 6.1.

Theorem 6.2 Let $M$ be a compact nearly Kähler 6-manifold, and $\mathcal{H}^{i}(M)$ the space of harmonic $i$-forms on $M$. Then $\mathcal{H}^{i}(M)$ is a direct sum of harmonic forms of pure Hodge type:

$$
\mathcal{H}^{i}(M)=\bigoplus_{i=p+q} \mathcal{H}^{p, q}(M)
$$

Moreover, $\mathcal{H}^{p, q}(M)=0$ unless $p=q$ or $(p=2, q=1)$ or $(q=1, p=2)$. 
Proof From Theorem 6.1, we find that a form $\eta$ is harmonic if and only if $\partial \eta=\bar{\partial} \eta=$ $\partial^{*} \eta=\bar{\partial}^{*} \eta=0$ and $\Delta_{N+\bar{N}} \eta=0$. From Corollary 5.2, we find that the latter equation is equivalent to $N \eta=\bar{N} \eta=N^{*} \eta=\bar{N}^{*} \eta=0$. We find that a form $\eta$ is harmonic if and only if all the Hodge components of $d, d^{*}$ vanish on $\eta$ :

$$
\partial \eta=\bar{\partial} \eta=\partial^{*} \eta=\bar{\partial}^{*} \eta=N \eta=\bar{N} \eta=N^{*} \eta=\bar{N}^{*} \eta=0 .
$$

Therefore, all Hodge components of $\eta$ also satisfy (6-4). This implies that these components are also harmonic. We proved (6-3).

To prove that $\mathcal{H}^{p, q}(M)$ vanishes unless $p=q$ or $(p=2, q=1)$ or $(q=1, p=2)$, we use Corollary 3.3. Let $\eta$ be a nonzero harmonic $(p, q)$-form. Then the scalar operator $R=\Delta_{\partial}-\Delta_{\bar{\partial}}$ vanishes on $\eta, R=\lambda^{2}(3-p-q)(p-q)$. Therefore, either $p=q$ or $p+q=3$. We obtain immediately that $p=q$ or $(p=2, q=1)$ or $(q=1, p=2)$ or $(p=3, q=0)$ or $(q=0, p=3)$. The last two cases are impossible: on $\Lambda^{3,0}(M)$, $\Lambda^{0,3}(M)$, the operator $N+\bar{N}$ is clearly injective (see (1-4)), hence it cannot vanish; however, by (6-4) we have $N+\bar{N}(\eta)=0$. This proves Theorem 6.2.

Remark 6.3 The middle cohomology of a compact nearly Kähler 6-manifold is remarkably similar to the middle cohomology of a Kähler manifold. In particular, the intermediate Jacobian $T:=H^{2,1}(M) / H^{3}(M, \mathbb{Z})$ is well defined in this case as well. As in the Kähler case, $T$ is a compact complex torus, and we have a pseudoholomorphic map $S \longrightarrow T$ from the space of pseudoholomorphic rational curves on $M$ to the intermediate Jacobian.

Remark 6.4 All harmonic forms $\eta \in \mathcal{H}^{p, q}(M),(p=2, q=1)$ or $(q=1, p=2)$ are primitive and coprimitive, that is, satisfy $L_{\omega}(\eta)=\Lambda_{\omega}(\eta)=0$. Indeed, $\{N, \bar{N}\}$ vanishes on $\eta$ as follows from (6-4). However, Proposition 3.2 implies that $\{N, \bar{N}\}(\eta)=$ $(p-q) \omega \wedge \eta$, hence $\omega \wedge \eta=0$.

Similarly, all harmonic forms $\eta \in \mathcal{H}^{p, p}(M)$ are primitive for $p=1$ and coprimitive for $p=2$. This is implied directly by (6-4) and the local expression for $N$ given in (1-4). For instance, for a $(1,1)$-form $\eta$, we have $N(\eta)=\Lambda_{\omega}(\eta) \cdot \Omega$, hence $\eta$ is primitive if $N(\eta)=0$.

\section{Appendix: Hodge theory on orbifolds}

In the appendix, we explain how the results of this paper can be applied to compact nearly Kähler orbifolds. 
Recall that an orbifold is a topological space equipped with an atlas of local charts, which are isomorphic to $\mathbb{R}^{n} / G$, where $G$ is a finite group acting faithfully and smoothly, and with all the gluing maps smooth and compatible with the group action.

The differential forms on an orbifold are defined in local charts as $G$-invariant differential forms on $\mathbb{R}^{n}$, which are compatible with the gluing maps.

The de Rham algebra and de Rham cohomology are defined literally in the same way as for manifolds, and they are equal to the singular cohomology. This was first observed by I Satake, who defined the orbifolds in 1950s and called them " $V$-manifolds" [27; 28]. Since Satake, all the usual constructions of smooth topology, such as the Chern-Weil theory of characteristic classes, Atiyah-Singer index formula, signature theorem and Riemann-Roch-Grothendieck theorem, were generalized for the orbifolds (see eg Kawasaki [18; 19; 20].

The Hodge theory identifies harmonic forms with the de Rham cohomology, using the closedness of the image of the de Rham differential on a compact manifold. The basic machinery here works for the orbifolds as well as in the smooth case [20].

The results of the present paper, such as Theorem 6.2, are obtained by application of local formulas for Laplacians to the global statements about cohomology, using the identification of cohomology and harmonic forms. These arguments are transferred to the orbifold case word by word.

The vanishing theorems, such as Theorem 6.1, are obtained by showing that a difference of certain second order operators is positive, which implies that a kernel of one of these operators lies inside a kernel of another. Here the vanishing arguments are also translated to the orbifold case, without any difficulty.

Acknowledgements I am grateful to Robert Bryant and Paul-Andi Nagy for enlightening correspondence. Nagy also suggested to add Remark 6.4.

Misha Verbitsky is partially supported by RFBR grant 10-01-93113-NCNIL-a, AG Laboratory SU-HSE, RF government grant, ag 11.G34.31.0023, Science Foundation of the SU-HSE award number 10-09-0015, and Simons-IUM fellowship.

\section{References}

[1] B S Acharya, J M Figueroa-O'Farrill, CM Hull, B Spence, Branes at conical singularities and holography, Adv. Theor. Math. Phys. 2 (1998) 1249-1286 (1999) MR1693624

[2] B S Acharya, E Witten, Chiral fermions from manifolds of $G_{2}$ holonomy arXiv: hep-th/0109152 
[3] M Atiyah, E Witten, $M$-theory dynamics on a manifold of $G_{2}$ holonomy, Adv. Theor. Math. Phys. 6 (2002) 1-106 MR1992874

[4] C Bär, Real Killing spinors and holonomy, Comm. Math. Phys. 154 (1993) 509-521 MR1224089

[5] H Baum, T Friedrich, R Grunewald, I Kath, Twistors and Killing spinors on Riemannian manifolds, Teubner-Texte zur Math. 124, Teubner Verlag, Stuttgart (1991) MR1164864 With German, French and Russian summaries

[6] J-B Butruille, Classification des variétés approximativement kähleriennes homogènes, Ann. Global Anal. Geom. 27 (2005) 201-225 MR2158165 In French

[7] R Cleyton, A Swann, Einstein metrics via intrinsic or parallel torsion, Math. Z. 247 (2004) 513-528 MR2114426

[8] J Eells, S Salamon, Twistorial construction of harmonic maps of surfaces into fourmanifolds, Ann. Scuola Norm. Sup. Pisa Cl. Sci. (4) 12 (1985) 589-640 (1986) MR848842

[9] T Friedrich, Nearly Kähler and nearly parallel $G_{2}$-structures on spheres, Arch. Math. (Brno) 42 (2006) 241-243 MR2322410

[10] T Friedrich, S Ivanov, Parallel spinors and connections with skew-symmetric torsion in string theory, Asian J. Math. 6 (2002) 303-335 MR1928632

[11] A Gray, Minimal varieties and almost Hermitian submanifolds, Michigan Math. J. 12 (1965) 273-287 MR0184185

[12] A Gray, Nearly Kähler manifolds, J. Differential Geometry 4 (1970) 283-309 MR0267502

[13] A Gray, Weak holonomy groups, Math. Z. 123 (1971) 290-300 MR0293537

[14] A Gray, The structure of nearly Kähler manifolds, Math. Ann. 223 (1976) 233-248 MR0417965

[15] P Griffiths, J Harris, Principles of algebraic geometry, Pure and Applied Math., Wiley-Interscience, New York (1978) MR507725

[16] N Hitchin, Stable forms and special metrics, from: "Global differential geometry: the mathematical legacy of Alfred Gray (Bilbao, 2000)", (M Fernández, J A Wolf, editors), Contemp. Math. 288, Amer. Math. Soc. (2001) 70-89 MR1871001

[17] S Ivanov, Connections with torsion, parallel spinors and geometry of Spin(7) manifolds, Math. Res. Lett. 11 (2004) 171-186 MR2067465

[18] T Kawasaki, The signature theorem for $V$-manifolds, Topology 17 (1978) 75-83 MR0474432

[19] T Kawasaki, The Riemann-Roch theorem for complex V-manifolds, Osaka J. Math. 16 (1979) 151-159 MR527023 
[20] T Kawasaki, The index of elliptic operators over V-manifolds, Nagoya Math. J. 84 (1981) 135-157 MR641150

[21] V F Kiričenko, K-spaces of maximal rank, Mat. Zametki 22 (1977) 465-476 MR0474103 In Russian

[22] S Kobayashi, K Nomizu, Foundations of differential geometry, Vol. II, Interscience Tracts in Pure and Applied Math. 15, Wiley-Interscience, New York-London-Sydney (1969) MR0238225

[23] A Moroianu, P-A Nagy, U Semmelmann, Unit Killing vector fields on nearly Kähler manifolds, Internat. J. Math. 16 (2005) 281-301 MR2130627

[24] A Moroianu, P-A Nagy, U Semmelmann, Deformations of nearly Kähler structures, Pacific J. Math. 235 (2008) 57-72 MR2379770

[25] O Muškarov, Structures presque hermitiennes sur des espaces twistoriels et leurs types, C. R. Acad. Sci. Paris Sér. I Math. 305 (1987) 307-309 MR910366 In French

[26] P-A Nagy, Nearly Kähler geometry and Riemannian foliations, Asian J. Math. 6 (2002) 481-504 MR1946344

[27] I Satake, On a generalization of the notion of manifold, Proc. Nat. Acad. Sci. U.S.A. 42 (1956) 359-363 MR0079769

[28] I Satake, The Gauss-Bonnet theorem for V-manifolds, J. Math. Soc. Japan 9 (1957) 464-492 MR0095520

[29] M Verbitsky, Hyper-Kähler manifolds with torsion, supersymmetry and Hodge theory, Asian J. Math. 6 (2002) 679-712 MR1958088

[30] M Verbitsky, Theorems on the vanishing of cohomology for locally conformally hyperKähler manifolds, Tr. Mat. Inst. Steklova 246 (2004) 64-91 MR2101284

[31] M Verbitsky, An intrinsic volume functional on almost complex 6-manifolds and nearly Kähler geometry, Pacific J. Math. 235 (2008) 323-344 MR2386227

Laboratory of Algebraic Geometry, Faculty of Mathematics, NRU HSE

7 Vavilova U1, Moscow 117312, Russia

verbit@mccme.ru, verbit@maths.gla.ac.uk

http://verbit.ru/

Proposed: Gang Tian

Received: 19 June 2008

Seconded: Simon Donaldson, Yasha Eliashberg

Revised: 7 October 2010 